\title{
TOPICS IN SPORTS MEDICINE
}

\section{A Young Female Athlete With Acute Low Back Pain Caused by Stage IV Breast Cancer

\author{
Evan A. Kahn, DC, DACBSP
}

\begin{abstract}
Objective: The purpose of this case report is to describe the case of a young female athlete with low back pain caused by metastatic breast cancer.

Clinical Features: A 27-year-old woman presented with low back pain after striking a ball during kickball 3 days earlier. Because of the mechanism of injury and onset, the patient was originally diagnosed with a lumbar spine sprain/ strain.

Intervention/Outcome: After radiographs were obtained and were read as unremarkable, a 2-week trial of care was initiated that included soft-tissue mobilizations, anti-inflammatory medications from her primary care physician, and therapeutic rehabilitation exercises. After this trial concluded, the patient did not improve and continued to be in significant pain. Magnetic resonance imaging was then ordered and revealed an expansile lesion at L2 with cortical compromise. Referral to an oncologist prompted the diagnosis of stage IV breast cancer.

Conclusion: Poor response to conservative treatment may indicate the working diagnosis is incorrect and that it must be reconsidered. In this case, a lack of response to care with persistent high severity of pain despite a multimodal approach justified further investigation with advanced imaging, which revealed spinal metastases secondary to breast cancer. Clinicians should be aware of history and physical exam indicators of red flag conditions that may present as low back pain. (J Chiropr Med 2017;16:230-235)

Key Indexing Terms: Breast Neoplasms; Female; Athletes; Fractures, Bone; Fractures, Spontaneous; Lumbar Vertebrae; Magnetic Resonance Imaging
\end{abstract}

\section{INTRODUCTION}

According to the Surveillance, Epidemiology, and End Results (SEER) Program, a National Institutes of Health program that tracks cancer statistics, an estimated 246600 new cases of breast cancer will be diagnosed in the United States in 2016. ${ }^{1}$ SEER also estimates that the incidence rates for 30-year-old US women being diagnosed with breast cancer range from $0.44 \%$ over 10 years to $4.05 \%$ over 30 years. Roughly $1.8 \%$ of all new cases of breast cancer are diagnosed between the ages of 20 and 34 .

Of all new diagnoses, $5.6 \%$ of de novo breast cancer diseases are initially found to be stage IV. ${ }^{2}$ A Swiss study of 254 breast cancer patients with bone metastases reported that 36 patients $(14.2 \%)$ with a median age of 62 had

Private practice, Holly Springs, North Carolina.

Corresponding Author: Evan A. Kahn, DC, DACBSP, 154 Village Walk Dr, Holly Springs, NC 27540. Tel.: +1 984-225-2149. (e-mail: evan.kahn@gmail.com).

Paper submitted October 3, 2016; in revised form June 14, 2017; accepted June 15, 2017.

1556-3707

(C) 2017 National University of Health Sciences.

http://dx.doi.org/10.1016/j.jcm.2017.06.002 developed a pathological fracture. The same study reported that in $40 \%$ of cases with pathological fracture, the diagnosis was bone metastasis. ${ }^{3}$

Low back pain is a considerably common condition. A systematic review by Hoy et al estimated the 1-year prevalence rate at $38 \%$ of the global population. ${ }^{4}$ They also estimated recurrence rates at the 1 -year point ranging from $24 \%$ to $80 \%$, with the highest rates of incidence tending to occur in the third decade of life and with prevalence increasing with age until 60 to 65 years. ${ }^{5}$ There are a myriad of risk factors including, but not limited to, working with heavy loads, manual tasks, lifting, awkward postures, low educational status, stress, anxiety, depression, and obesity. ${ }^{6}$

The purpose of this article is to describe a young healthy female athlete with lower back pain presenting with a pathological fracture at L2 secondary to metastatic breast cancer.

\section{CASE RePORT}

A 27-year-old white woman presented to a chiropractic office with the chief complaint of lower back pain after warming up and participating in a kickball match. She reported she felt some discomfort while she was jogging, 
but subsequently had pain after striking the ball with her right foot. She reported pain at the L2/3 level with mild radiation into the right paravertebral region. She described the pain as constant, sharp, and stabbing and rated it as an 8/ 10 on a numeric pain scale. She denied any radiating pain or paresthesias into the extremities. She had a history of uncomplicated low back pain that was previously relieved with conservative care at the same office. This history dated back to her teenage years and was attributed to a mild lumbar scoliosis.

The patient's physical exam revealed a decrease in flexion and extension active range of motion with pain in the upper lumbar area. Kemp's and Yeoman's tests were positive for localized pain. She demonstrated +4 tenderness to palpation at the L2 level. Patellar and Achilles deep tendon reflexes were 2/4 bilaterally. Babinski and lower extremity clonus were absent bilaterally. Lower extremity myotomal testing was $5 / 5$ bilaterally. Increased paraspinal muscle tone was noted at the level of complaint. The patient had a family history of breast cancer. Her aunt and grandmother were diagnosed in their $50 \mathrm{~s}$ and $60 \mathrm{~s}$, respectively. In years past she had routine mammograms to observe a cyst in her right breast. At the time of this incident, she had not been screened within the past year.

The patient was diagnosed with a lumbar spine sprain/ strain with other differential diagnoses including discogenic pain and spondylolysis. Radiographs obtained to rule out spondylolysis did not reveal signs of fracture or dislocation; however, in retrospect, subtle lucencies are visible in the vertebral body and left pedicle (Fig 1). Treatment was then rendered and included soft tissue mobilizations to the lumbar spine and hips.

The patient was intolerant to side posture positioning for manipulation, so the procedure was not performed. The patient was placed on a conservative treatment plan for 2 weeks.

The patient also reported seeing her employer's on-site physician, who prescribed her with oral anti-inflammatory drugs and physical therapy. Physical therapy sessions were directed to strengthen core musculature and increase range of motion.

Roughly 2 weeks later, the patient returned to the chiropractic office with no change in signs or symptoms. A magnetic resonance imaging (MRI) scan of her lumbar spine was ordered to rule out stress edema and/or discogenic pathology. The MR image was read by a radiologist and found to have significant findings of a 2.2-cm expansile lesion in the left L2 vertebral body with associated overlying vertebral superior endplate concavity focally (Figs 2 and 3). The extension of the mass into the pedicle and obvious cortical expansion/destruction along the lateral side of the vertebral body created a differential diagnosis of hemangioma with pathologic fracture, giant cell tumor, osteoblastoma, plasmacytoma, or metastatic disease of unknown origin. There was also a subtle $\mathrm{T} 2$ hyperintense lesion along the superior endplate of L1 eccentric to the right that represented a second lesion (not pictured here), increasing the chances of a malignant diagnosis.

The patient was subsequently referred to an oncologist, who arrived at a diagnosis of stage IV breast carcinoma. A mammogram and ultrasound-guided biopsy revealed a cancerous tumor in the right breast. Two more metastatic

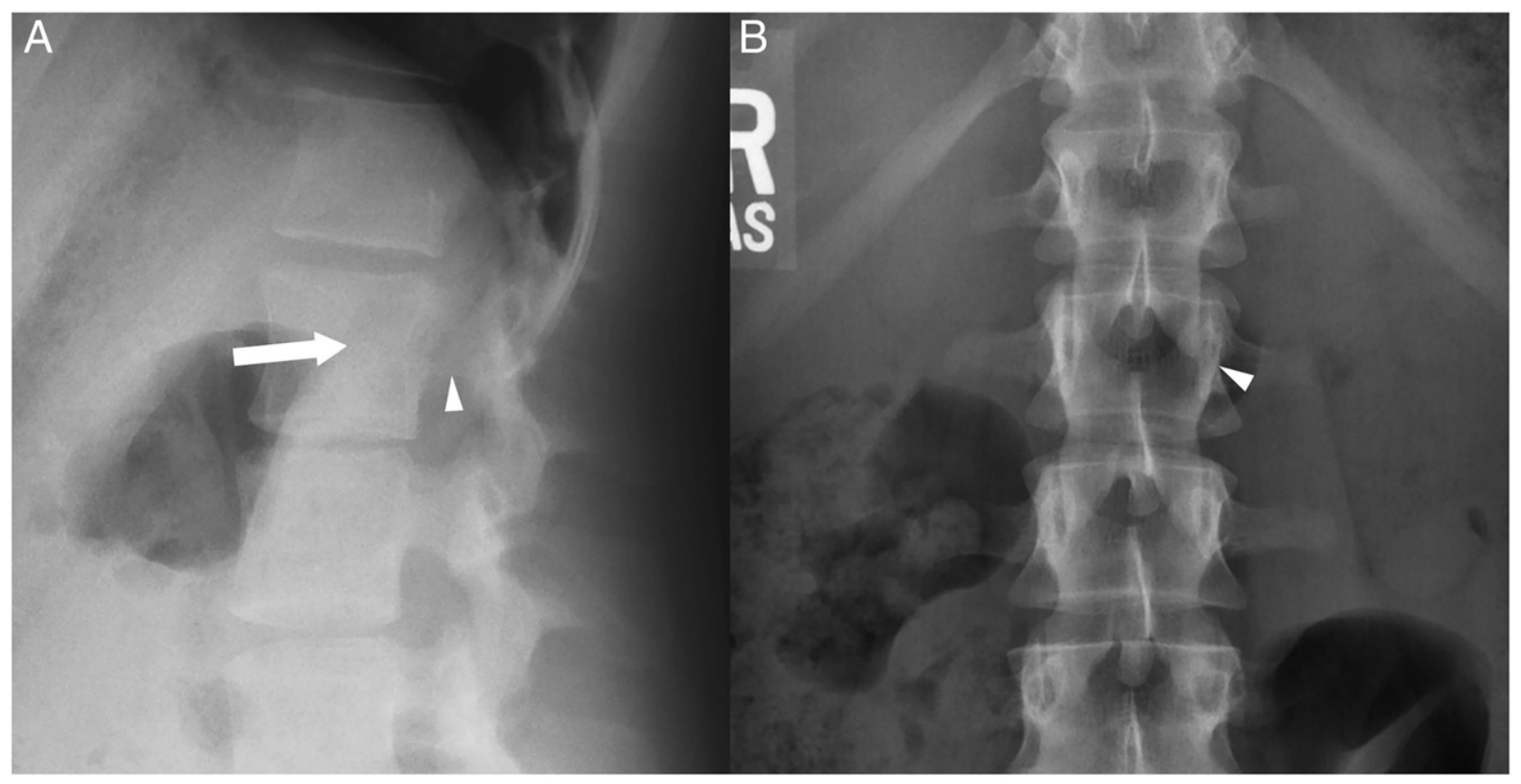

Fig I. (A) Lateral and (B) anteroposterior thoracolumbar radiographs demonstrating subtle lucencies within the vertebral body (arrow) and left pedicle (arrowheads) of L2. 

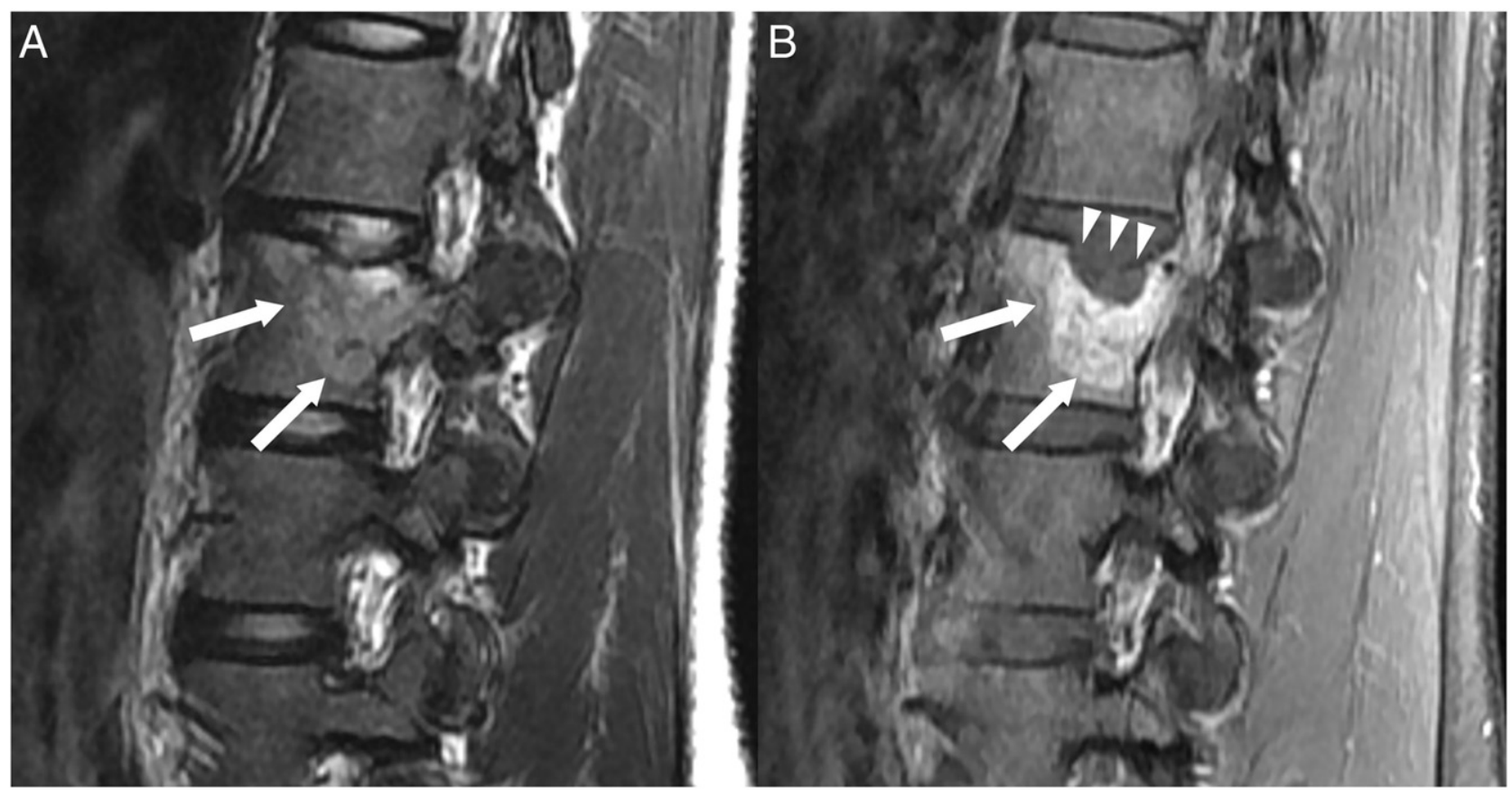

Fig 2. (A) Sagittal T2-weighted and (B) sagittal T1-weighted, fat-suppressed (with contrast) magnetic resonance imaging scans revealing the L2 vertebral body lesion (arrows) with destruction of the superior endplate of L2 (arrowheads).

lesions were later discovered at the $\mathrm{C} 6$ and $\mathrm{C} 7$ spinal levels via bone scan and MRI.

The patient was diagnosed in 2014 with the hormone receptor-positive/human epidermal growth factor receptor 2-negative (HR+/HER2-) subtype, the most common form of breast cancer. The cyst in question was not the primary lesion and was found to be benign; however, there was a previously unseen lesion behind the original cyst, which proved malignant.

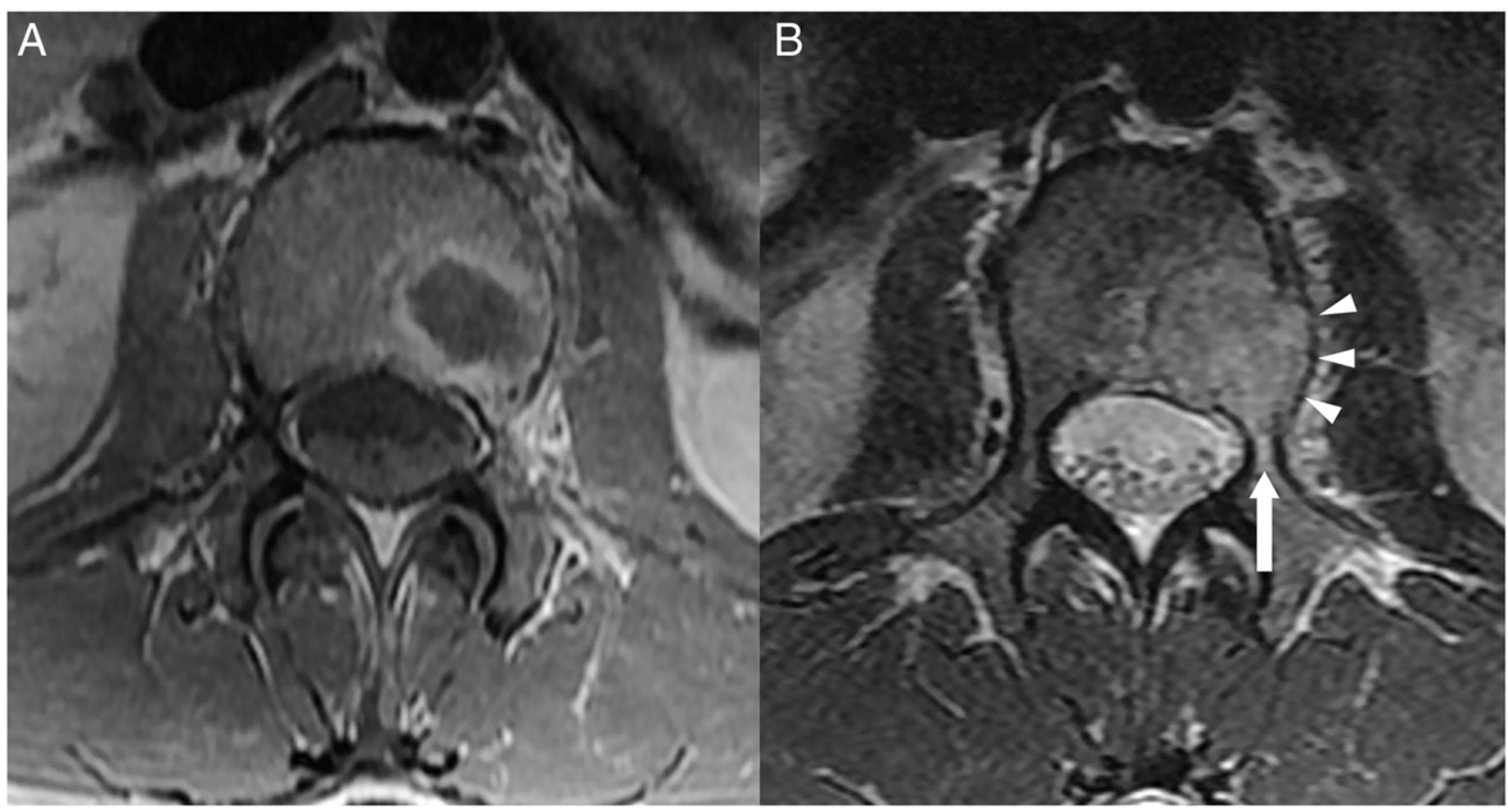

Fig 3. (A) Axial T1-weighted and (B) axial T2-weighted magnetic resonance imaging scans revealing the L2 vertebral body lesion with extension into the left pedicle (arrow) and osseous expansion (arrowheads). 
After her diagnoses, she was initially placed on injections of Letrozole, an estrogen production inhibitor, and Xgeva, a therapy used to inhibit the acceleration of osteoclastic activity in bone tumors. She was later placed on Ibrance, a cyclin-dependent kinase 4 and 6 (CDK4/6) inhibitor, and Faslodex, an estrogen receptor blocker.

In 2016, she underwent a successful lumpectomy of her right breast, where 6 lymph nodes were removed from her axillary region. As of this writing, her spinal lesions have totally diminished through the use of oral hormonal drugs and chemotherapy drugs. However, a lesion on her liver was recently recognized on routine imaging and, through biopsy, was confirmed cancerous.

In 2017, she began a trial of Xeloda, an antimetabolite cell cycle-specific chemotherapy drug designed to inhibit cell division. Throughout her recovery she was periodically administered the Xgeva injections and continues to receive them to this day. She is still currently active in exercise, likes to participate in weight training and bowling, and coaches grade-school basketball. The patient provided consent to publish this case report.

\section{DISCUSSION}

According to the SEER Cancer Statistics Review, breast cancer is discovered in white women aged 25 to 29 at a rate of 8.8 for every $100000 .^{7}$ The spinal column is the most common osseous location for metastatic deposits, ${ }^{8}$ whereas metastases from breast cancer that locate to the spine occur at a $74.3 \%$ rate, second highest only to prostate cancer. ${ }^{9}$ The thoracic spine is the most common site for metastatic disease (70\%), followed by the lumbar spine $(20 \%)$ and the cervical spine $(10 \%) .{ }^{10}$ The vertebral column is the most commonly afflicted portion $(85 \%)$; the paravertebral region $(10 \%-15 \%)$ and epidural or subarachnoid/intramedullary space $(<5 \%)$ are more rarely involved.

Breast cancer is staged using the American Joint Committee on Cancer TNM system, which is based on the size of the tumor, whether the cancer has reached nearby lymph nodes, and/or whether the cancer has metastasized. ${ }^{11}$ If the cancer has metastasized to distant organs or lymph nodes far from the breast, it is considered stage IV regardless of size or spread to nearby lymph nodes.

The patient's diagnosis was ultimately discovered through a pathological fracture of her lumbar spine caused by a metastatic lesion originating from the breast. Her case did not provide any obvious red flags. However, a familial history of breast cancer, a lack of response to care, and continued high severity of pain despite a multimodal approach are appropriate justifications for ordering advanced imaging.

Frequent indicators from a patient's history that low back pain may not be mechanical in nature include an absence of a mechanism of injury; insidious onset; flank pain; associated abdominal pain or gastrointestinal distress; incontinence; and saddle paresthesia. Indicators from the patient's physical exam that low back pain may not be mechanical in nature include an inability to reproduce the patient's complaint through orthopedic testing; a positive Valsalva maneuver; presence of fever; neurological findings such as hyporeflexia, hypoesthesia, or myotomal weakness; and abdominal palpation revealing referral pain to the low back. A combination of the prior signs and symptoms suggests nonmechanical low back pain and may require further investigation.

Red flag markers in a patient's history or physical exam continue to be important when searching for malignancy or fracture. The red flags that have proven to be of the most value when screening for fracture in a low back pain patient are older age, prolonged use of corticosteroids, severe trauma, and presence of a contusion or abrasion. The red flag with the highest probability of spinal malignancy is prior history of malignancy. ${ }^{12}$

Currently, immediate or routine lumbar spine radiography is not suggested without indications of serious underlying pathology. ${ }^{13}$ It is important to note that $30 \%$ to $50 \%$ of osseous destruction is necessary for lytic lesions

Table I. ACR Appropriateness Criteria-Acute, Subacute, Chronic Uncomplicated Low Back Pain or Radiculopathy; No Red Flags; No Prior Management

\begin{tabular}{lll}
\hline Radiologic Procedure & Rating $^{\text {a }}$ & Comments \\
\hline MRI lumbar spine without IV contrast & 2 & \\
X-Ray lumbar spine & 2 & \\
X-Ray myelography and postmyelography CT lumbar spine & 2 & If there is concern for spondylolysis in a young patient, \\
$99 \mathrm{~m}$ Tc bone scan with SPECT spine & 2 & SPECT/CT remains the gold standard. \\
& 2 & \\
CT lumbar spine without IV contrast & 2 & \\
CT lumbar spine with IV contrast & 2 & \\
MRI lumbar spine without and with IV contrast & 1 & \\
CT lumbar spine without and with IV contrast & & \\
\hline
\end{tabular}

$A C R$, American College of Radiology; $C T$, computed tomography; $I V$, intravenous; $M R I$, magnetic resonance imaging; SPECT, single-photon emission computed tomography; ${ }^{99 \mathrm{~m}} \mathrm{Tc}$, technetium-99m.

Adapted from the 2017 ACR Appropriateness Criteria Ratings for Low Back Pain. Reston, VA: American College of Radiology. Reprinted with permission.

${ }^{\text {a }}$ Rating scale: 1-3, usually not appropriate; 4-6, may be appropriate; 7-9, usually appropriate. 
Table 2. ACR Appropriateness Criteria-Acute, Subacute, or Chronic Uncomplicated Low Back Pain or Radiculopathy-One or More of the Following: Low-Velocity Trauma, Osteoporosis, Elderly Individual, or Chronic Steroid Use

\begin{tabular}{|c|c|c|}
\hline Radiologic Procedure & Rating $^{a}$ & Comments \\
\hline X-Ray lumbar spine & 7 & $\begin{array}{l}\text { This procedure is recommended as the initial imaging study, } \\
\text { especially in patients with osteoporosis or history of steroid use. }\end{array}$ \\
\hline CT lumbar spine without IV contrast & 7 & $\begin{array}{l}\text { If there remains concern over vertebral body fracture, } \\
\text { detailed osseous analysis with CT can be performed for further evaluation. }\end{array}$ \\
\hline MRI lumbar spine without IV contrast & 7 & $\begin{array}{l}\text { CT is preferred. MRI can be useful to evaluate for ligamentous } \\
\text { injury or worsening neurologic deficit. MRI can depict marrow } \\
\text { edema in these scenarios. }\end{array}$ \\
\hline${ }^{99 \mathrm{~m}} \mathrm{Tc}$ bone scan with SPECT spine & 3 & $\begin{array}{l}\text { Bone scan with SPECT/CT can be useful for radiographically } \\
\text { occult fractures and problem solving. }\end{array}$ \\
\hline CT lumbar spine with IV contrast & 3 & \\
\hline CT lumbar spine without and with IV contrast & 1 & \\
\hline $\mathrm{X}$-Ray myelography and postmyelography CT lumbar spine & 1 & \\
\hline X-Ray discography and postdiscography CT lumbar spine & 1 & \\
\hline
\end{tabular}

$A C R$, American College of Radiology; $C T$, computed tomography; $I V$, intravenous; $M R I$, magnetic resonance imaging; SPECT, single-photon emission computed tomography; ${ }^{99 \mathrm{~m}} \mathrm{Tc}$, technetium- $99 \mathrm{~m}$.

Adapted from the 2017 ACR Appropriateness Criteria Ratings for Low Back Pain. Reston, VA: American College of Radiology. Reprinted with permission.

${ }^{\text {a }}$ Rating scale: 1-3, usually not appropriate; 4-6, may be appropriate; 7-9, usually appropriate.

to show up on radiographs. ${ }^{14}$ The detection of a given metastatic lesion using conventional radiography also depends on a number of other factors including the size of the lesion, the location within the bone, and the effect of the lesion on the cortex or surrounding trabeculae. Clinical indications for advanced imaging in adults with low back pain are based on suspicion of cancer, spinal infection, fracture even when X-rays are negative, inflammatory back pain, severe or progressive neurological deficit, severe or progressive low back pain, and subacute or chronic low back pain with radiculopathy that does not respond to conservative care. ${ }^{15}$

The American College of Radiology's (ACR) Appropriateness Criteria Ratings provide guidelines on when it is appropriate to order imaging for low back pain. ${ }^{16}$ The ACR guidelines base the modality on a numerical rating scale from 1 to 9, where 1-3 indicate usually not appropriate, 4-6 indicate may be appropriate, and 7-9 indicate usually appropriate. Imaging modalities for variants of low back pain are organized and respectively graded. Lumbar spine radiographs and MRI without contrast for acute low back pain with no red flags or prior management receive a rating of 2, or usually not appropriate (Table 1). Lumbar spine radiographs and MRI without contrast for acute low back pain as a result of low-velocity trauma receive a rating of 7 , or usually appropriate (Table 2). In this patient's case, initial radiographs would be considered appropriate, as was a follow-up MRI 3 weeks later.

The patient was placed on Ibrance, an oral medication that acts as a CDK4/6 inhibitor that specifically targets and slows cellular growth. According to Ingham and Schwartz, CDK4/6 inhibitors are the most promising form of cell-cycle therapeutics to date. ${ }^{17}$ Yoon et al performed a retrospective analysis from 1989 to 2008, investigating the outcomes in younger (under 35) and older (over 35) patients with metastatic breast cancer. ${ }^{18}$ They found that the younger group who had the subtype HR+/HER2- had a significantly improved disease-free survival rate as opposed to the HR+ type tumor alone, which had a significantly poorer disease-free survival rate.

\section{Limitations}

Case studies are inherently limited as they describe 1 case managed by 1 clinician. Some aspects of the case are potentially biased depending on the clinician's perception of how the case progressed. Also, the author did not have access to the medical doctor's or the physical therapist's records who were treating the patient at the same time as the clinician.

\section{CONCLUSION}

This case illustrates the importance of ordering advanced imaging timely and when appropriate. Low back pain that fails to respond over time and to multi-modal conservative care could be considered as a red flag and must be re-evaluated for pathology. Metastatic breast cancer and subsequent pathological fracture is extremely rare in young adults but should be considered when a familial history is present and relatively benign actions cause significant pain.

\section{Funding Sources ANd Conflicts of INTEREST}

No funding sources or conflicts of interest were reported for this study. 


\section{CONTRIBUTORSHIP INFORMATION}

Concept development (provided idea for the research):

E.K.

Design (planned the methods to generate the results):

E.K.

Supervision (provided oversight, responsible for organization and implementation, writing of the manuscript): E.K.

Data collection/processing (responsible for experiments, patient management, organization, or reporting data): E.K.

Analysis/interpretation (responsible for statistical analysis, evaluation, and presentation of the results): E.K.

Literature search (performed the literature search): E.K. Writing (responsible for writing a substantive part of the manuscript): E.K.

Critical review (revised manuscript for intellectual content, this does not relate to spelling and grammar checking): E.K.

\section{Practical Applications}

- Physicians must be aware of all possible diagnoses, especially when conservative options fail.

- Red flag signs/symptoms are highlighted.

- This case brings to light a challenging diagnosis in a young female athlete.

\section{REFERENCES}

1. Howlader N, Noone AM, Krapcho M, et al. SEER Cancer Statistics Review, 1975-2012. Bethesda MD: National Cancer Institute; 2015.
2. Breast cancer, stage distribution of SEER incidence cases, 2005-2014, by race/ethnicity. National Cancer Institute, Surveillance Research; 2015. Available at: https://seer. cancer.gov/statfacts $/ \mathrm{html} /$ breast.html. Accessed September 15, 2017.

3. Vetter M, Huang DJ, Güth U. Pathological fractures in breast cancer patients with bone metastases. Ann Oncol. 2014;25(4):116-136.

4. Hoy D, Bain C, Williams G, et al. A systematic review of the global prevalence of low back pain. Arthritis Rheum. 2012; 64(6):2028-2037.

5. Hoy D, Brooks P, Blyth F, Buchbinder R. The epidemiology of low back pain. Best Pract Res Clin Rheumatol. 2010;24(6):769-781.

6. Oliveira VC, Ferreira ML, Refshauge KM, et al. Risk factors for low back pain: insights from a novel case-control twin study. Spine J. 2015;15(1):50-57.

7. Breast cancer, SEER incidence rates by age at diagnosis, 2010-2014, by race/ethnicity. SEER Cancer Statistics; 2016. Available at: https://seer.cancer.gov/statfacts/html/breast. html. Accessed September 15, 2017.

8. Böhm P, Huber J. The surgical treatment of bony metastases of the spine and limbs. J Bone Joint Surg Br. 2002;84(4):521-529.

9. Wong DA, Fornasier VL, MacNab I. Spinal metastases: the obvious, the occult, and the imposters. Spine (Phila Pa 1976). 1990;15(1):1-4.

10. Klimo Jr P, Schmidt MH. Surgical management of spinal metastases. Oncologist. 2004;9(2):188-196.

11. American Joint Committee on Cancer. Breast. In: Edge S, Byrd DR, Compton CC, Fritz AG, Greene F, Trotti A, eds. AJCC Cancer Staging Manual. 7th ed. New York: Springer; 2010. p. 347-369.

12. Downie A, Williams CM, Henschke N, et al. Red flags to screen for malignancy and fracture in patients with low back pain: systematic review. BMJ. 2013;347:f7095.

13. Chou R, Fu R, Carrino JA, Deyo RA. Imaging strategies for low-back pain: systematic review and meta-analysis. Lancet. 2009;373(9662):463-472.

14. Harrington KD. Metastatic disease of the spine. J Bone Joint Surg Am. 1986;68(7):1110-1115.

15. Millán OE, Cabrera A, Muñiz SJ, Sola SC, Zubia AJ. Indications for magnetic resonance imaging for low back pain in adults. Rev Calid Asist. 2014;29(1):51-57.

16. Patel ND, Broderick DF, Burns J, et al. ACR Appropriateness Criteria ${ }^{\circledR}$ : Low Back Pain. Available at: https://acsearch.acr. org/docs/69483/Narrative/. Accessed November 20, 2016.

17. Ingham M, Schwartz GK. Cell-cycle therapeutics come of age. J Clin Oncol. 2017;35(25):2949-2959.

18. Yoon TI, Hwang UK, Kim ET, et al. Survival improvement in hormone-responsive young breast cancer patients with endocrine therapy. Breast Cancer Res Treat. 2017;165(2):311-320. 